\title{
L'art roman des Pyrénées : matériaux, technique et couleurs
}

Compte rendu et participation à un séminaire de l'université de Barcelone, 29-30 novembre 2007

Juliette Rollier-Hanselmann

\section{(2) OpenEdition}

Journals

Édition électronique

URL : https://journals.openedition.org/cem/7032

DOI : $10.4000 /$ cem.7032

ISSN : 1954-3093

Éditeur

Centre d'études médiévales Saint-Germain d'Auxerre

Édition imprimée

Date de publication : 15 août 2008

ISSN : 1623-5770

Référence électronique

Juliette Rollier-Hanselmann, «L'art roman des Pyrénées : matériaux, technique et couleurs », Bulletin du centre d'études médiévales d'Auxerre / BUCEMA [En ligne], 12 | 2008, mis en ligne le 01 juillet 2008, consulté le 22 septembre 2022. URL : http://journals.openedition.org/cem/7032 ; DOI : https://doi.org/ $10.4000 /$ cem.7032

Ce document a été généré automatiquement le 22 septembre 2022.

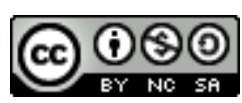

Creative Commons - Attribution - Pas d'Utilisation Commerciale - Partage dans les Mêmes Conditions 4.0 International - CC BY-NC-SA 4.0

https://creativecommons.org/licenses/by-nc-sa/4.0/ 


\title{
L'art roman des Pyrénées : matériaux, technique et couleurs
}

\author{
Compte rendu et participation à un séminaire de l'université de
} Barcelone, 29-30 novembre 2007

Juliette Rollier-Hanselmann

1 L'architecture romane des régions pyrénéennes a été analysée sous différents points de vue: formel, stylistique, structurel, historique et conceptuel. Pour comprendre les relations entre constructions, vie sociale et économique de l'époque, l'un des axes de recherche peut être celui de l'analyse des matériaux constitutifs des bâtiments, de leur qualité et leur variété.

2 Le séminaire, organisé par Marius Vendrell, professeur à l'université de Barcelone, clôturait un programme de recherches mené sur deux ans et qui réunissait des chercheurs espagnols et français. Ces derniers étaient représentés par Floréal Daniel, ingénieur de recherche à l'université de Bordeaux-3 [Institut de recherche sur les archéomatériaux - Centre de recherche en physique appliquée à l'archéologie, IRAMAT-CRPAA].

3 Un site internet a été créé, qui permet de visualiser toutes les conférences avec de nombreuses illustrations des sites («http://161.116.85.21/medieval )).

Étude des techniques de construction et des matériaux

4 Les nécessités de construction des édifices romans ont stimulé l'imagination des constructeurs qui ont trouvé de multiples solutions (J.Gonzales). Un programme de gestion et de documentation du patrimoine archéologique et architectural a été mis en place pour faire avancer la recherche (www.veclus.cat).

5 Les techniques de constructions romanes présentent certaines particularités régionales comme le montage des murs au moyen de "cuny »- assises de forme trapézoïdale. Ce procédé facilitait beaucoup la mise en place des parements romans (R. Gonzales et F. Caballé).

6 L'étude des matériaux, répertoriés sur des relevés d'élévation, permet ainsi de connaître les phases de construction d'un édifice (P. Giraldes). 
7 La finition des murs extérieurs est très variée selon les bâtiments, allant du joint incisé ou en relief, jusqu'aux polychromies les plus diverses (J.Camps). Il est important de documenter les vestiges qui subsistent et qui deviennent de plus en plus rares.

Dans certains cas les observations de terrain ont été complétées par des analyses de laboratoire (A. Lluveras).

Techniques picturales et restauration des peintures murales

De nombreux efforts ont été mis en œuvre pour restaurer et valoriser les peintures romanes des Pyrénées. Un panorama général a été présenté par P. Rovira, avec de nombreux exemples : S. Tomas de Fluvia, S. Maria de Montmelo, S. Victoria de Sauleda, S. Maria de Barbera, S. Maria de Mur, etc.

Les peintures murales de Saint-Clément de Tahull ont fait l'objet de différentes campagnes de restauration, allant des déposes des années 1920 jusqu'aux travaux récents (M. Marquès), en passant par la réalisation de copies.

11 Afin de mieux imaginer la polychromie intérieure d'un tel édifice, la restitution en image 3D s'avère particulièrement utile. Marius Vendrell a ainsi proposé des reconstitutions virtuelles de S.Maria de Tahull, ainsi que divers exemples de polychromies extérieures dont il subsiste encore des traces sur des édifices de la région. De même, à l'occasion de la restauration des peintures murales romanes de l'église de Vals (Ariège, XII ${ }^{e}$ siècle), une restitution 3D de l'abside de l'église et des peintures de la voûte a permis d'associer à l'image virtuelle toutes les informations, notamment physico-chimiques et iconographiques, recueillies sur ces peintures (P. Mora, Archéotransfert, université de Bordeaux-3). Il s'agit donc d'une base de données, couplée à une imagerie virtuelle, dont le modèle est capable d'évoluer en fonction de nouveaux acquis fournis par les différentes disciplines impliquées dans ce projet.

12 Une analyse très détaillée des techniques picturales a été réalisée pour S. Juan de Bohi (C. Payas), avec des relevés à partir des déposes du musée national d'Art catalan, ce qui permet de comprendre le processus d'élaboration pictural et de mieux connaitre les spécificités de cet atelier.

13 Les techniques de peintures murales en Aragon ont été abordées avec les exemples de S. Juan de Ruesta (peintures murales déposées) et S. Vincent et $\mathrm{S}$. Valero de Roda (J. Pérez). Les palettes de couleurs sont identiques : oxydes de fer, terre verte et noir de charbon. Le bleu est obtenu à partir d'un mélange de terre verte et de grains bleus d'aérinite, pigment spécifiquement pyrénéen, dont les mines se trouvent près de Ruesta.

14 Les peintures gothiques ont été évoquées avec l'exemple de La Seu Vella de Lérida (E. Balasch), en tenant compte de la complémentarité avec les polychromies sur pierre et les vitraux.

15 La restauration des peintures de $\mathrm{S}$. Valero (XIII ${ }^{\mathrm{e}}$ siècle) illustre bien la nécessité de documenter de façon complète les travaux (J. R. Gracia et J. L. Abad). La compréhension des causes d'altération permet ainsi de mieux prévoir les traitements appropriés.

Relation entre couleur et iconographie

16 L'équipe de l'université Bordeaux-3, dirigée par Floréal Daniel, propose d'aborder les problématiques d'interprétation des peintures romanes de façon transversale, en cherchant notamment la relation qui pourrait exister entre le choix des matériaux et l'iconographie. L'étude technique complète comprend la recherche documentaire, des 
relevés de l'état réel des œuvres, des analyses d'enduits et de pigments, en passant par l'étude des altérations chimiques et biologiques des matériaux.

17 L'analyse iconographique et technique des trois absides de S. Maria de Barbera del Vallès (B. Laborde) a fourni des nombreuses informations quant aux pratiques d'un chantier important du milieu du XII ${ }^{\mathrm{e}}$ siècle. La technique mixte et les matériaux ont été analysés de différentes façons (loupe, SEM, IRTF). La palette comprend le blanc de chaux, les oxydes de fer, le lapis-lazuli, la terre verte et le noir de charbon. Des matériaux d'altération et de restauration sont perceptibles par la présence d'oxalates et de stéarates de chaux.

La relation entre le choix des matériaux et la disposition des éléments du programme iconographique (A. Mounier) a été évoquée au moyen de quatre exemples français: Saint-Aventin et Notre-Dame de Vals (Haute-Garonne), Nogaro (Gers) et Moissac (chapelle abbatiale). Des comparaisons entre les palettes de couleurs permettent de constater l'usage de trois types de pigment bleu (aérinite, azurite et lapis-lazuli), ainsi que des dorures (Moissac). Cette grande variété des bleus est d'ailleurs une spécificité des sites pyrénéens, tant du côté français qu'espagnol.

L'analyse des pigments indique une grande diversité des matériaux mis en œuvre dans les édifices retirés des montagnes, comme Saint-Vincent d'Estamariu dans le Haut Urgell (S. Boularand). À S. Pere de Terrassa, c'est même le bleu égyptien qui a été utilisé, probablement à partir d'un remploi antique.

La réflexion autour des peintures de S. Juan de Bohi et des Salles-Lavauguyon permet de relancer la discussion sur les échanges qui ont eu lieu entre les ateliers français et espagnols (C. Voyer). Si certains sujets iconographiques de S. Juan ont pu être inspirés par la peinture clunisienne, le transfert des influences s'est également opéré par l'activité des scriptoria, notamment celui de Limoges.

L'étude stratigraphique de l'abside de la Chapelle-des-Moines de Berzé-la-Ville (J. Rollier) apporte des indications quant au type de matériaux utilisés et l'étendue des repeints qui cache en partie la peinture romane. Le raffinement technique est perceptible de différentes façons, notamment dans l'utilisation différenciée du bleu de lapis-lazuli selon les niveaux de lecture iconographique.

Diffusion des connaissances et vulgarisation auprès du public

En Catalogne, un effort considérable a été accompli pour rendre accessible les connaissances des spécialistes à un plus vaste public. En plus des instances patrimoniales officielles, qui œuvrent depuis longtemps, c'est un réseau de bénévoles, réuni par J. A. Olaneta, qui a publié de nombreux fascicules et ouvrages concernant les édifices des Pyrénées. Ce travail accélère la prise de conscience collective envers la richesse et de la fragilité du patrimoine régional.

Conclusion

23 La région pyrénéenne constitue un champ d'investigations particulièrement riche et intéressant. Les relations historiques qui lient la Catalogne, l'Aragon aux régions de l'ouest de la France, expliquent certains transferts de connaissances. Le chercheur est ainsi amené à analyser un bâtiment ou une peinture selon des angles très diversifiés. Il existe ainsi des matériaux intérieurs et extérieurs qui intègrent des décors variés.

Si la polychromie naturelle, obtenue par la variété de pierres est souvent conservée, les rares restes peints qui subsistent à l'extérieur sont fortement exposés aux intempéries et doivent être répertoriés. 
26 Au moment des restaurations, il est primordial d'avoir une connaissance complète de l'œuvre, afin de préconiser les meilleures solutions. La collaboration entre les différents intervenants s'avère primordiale. La nécessité d'une période d'étude et de réflexion permet d'engager avec sagesse toute intervention moderne sur ce patrimoine ancien fragile.

La dispersion des œuvres dans différentes régions nécessite un programme national et global visant à la préservation de ces témoins uniques de l'histoire.

La diffusion des connaissances auprès d'un large public s'avère très utile pour développer une prise de conscience collective. Les administrations devraient prévoir des actions pédagogiques à tous les niveaux, car chacun est concerné par le patrimoine de son pays.

D'une façon générale, il faut sensibiliser tous les acteurs politiques quant à l'importance du sauvetage des édifices romans qui font partie de l'histoire nationale. Programmedu jeudi 29 novembre José Luis GONZÁLEZ, L'arquitectura romànica de muntanya: solucions constructives i necessitats Reinald GONZÁLEZ, Francesc CABALLÉ, Els acabats de l'arquitectura medieval Pere ROVIRA, Conservació, seguiment i valoració de la restauració de pintures murals de la muntanya osefina PÉREZ, Materiales y técnicas de la pintura románica aragonesa: algunos ejemplos en el marco de los Pirineos Aurélie MOUNIER, Techniques et matériaux de la peinture en relation avec la disposition, dans l'architecture, des éléments du programme iconographique Esther BALASCH, Arquitectura de pinzell a les pintures murals de la Seu Vella de Lleida Mercè MARQUÈs, La campanya d'arrencament dels frescos medievals i la restauració/ reproducció dels conjunts murals a la vall de Boi en els darrers 50 anys Màrius VENDRELL, Els acabats de l'arquitectura religiosa romànica : l'exemple de Santa Maria de Taüll, restitució virtual Pilar GIRÁLDEZ, Aproximació a l'estudi dels materials dels edificis romànics dels Pirineus Floreal DANIEL, Uniformité des matériaux, diversité de destins. La vie mouvementée des peintures romanes à travers quelques exemples pyrénéens du mardi 30 novembre Juliette ROLLIER, Berzé-la-Ville, la Chapelle-des-Moines (XII ${ }^{e}$ siècle) : découverte d'un Christ caché sous les repeints

41 José RAMON GARCIA, José Luis ABAD, Proceso de restauración de las pinturas murales de la Cripta del Tesoro o de San Valero (s. XIII) y la capilla de la Infermería o de San Agustín (s. XV) de Roda de Isábena

42 Anna LLUVERAS, Anàlisi de materials pictòrics orgànics : possibilitats de les tècniques acoblades a espectrometria de masses

43 Sarah BOULARAND, Materials i tècniques de la pintura romànica de muntanya : l'exemple d'Estamariu

Bàrbara LABORDE, Les peintures murales de «La romànica » de Sta. Maria de Barberà : matériaux et technique picturale 
Cécile VOYER, Territoires et identités : la créativité artistique aux XI ${ }^{\mathrm{e}}-\mathrm{XII}^{\mathrm{e}}$ siècles

Jordi CAMPS, Sòria Materials i colors en l'arquitectura romànica de Catalunya

Clara PAYÀs, Construcció de les figures i sistemes de representació en la pinturamural romànica a partir de l'estudi de conjunt de Sant Joan de Boí

Juan Antonio OLAÑETA, La participación de las organizaciones civiles en el estudio, difusión y protección del patrimonio románico : la asociación de Amigos del Románico.

Pascal MORA, Restitution 3D et base de données (sur la conservation, les altérations, les analyses physico-chimiques) appliquée aux peintures de Vals (Ariège, XII ${ }^{e}$ siècle).

Taula rodona : conclusions

Participants - Ponents

José Luis GONZÁLEZ, Departament de Construccions Arquitectòniques, ETSAB Universitat Politècnica de Catalunya

Reinald GonZÁlez, Francesc Caballé Veclus, s. l. Gestió i documentació del patrimoni arquitectònic $\mathrm{i}$ arqueològic

Pere Rovira, Centre de Restauració de Bens Mobles de Catalunya, Departament de Cultura Generalitat de Catalunya

Josefina PÉREZ, Departamento de Química Analítica, universidad de Zaragoza

Aurélie MOUNIER, Institut de recherche sur les archéomatériaux (IRAMAT), UMR/CNRS/

Centre de recherche en physique appliquée à l'archéologie (CRPAA), université Bordeaux-3

Esther BALASCH, Museu Diocesà i Comarcal de Lleida

Mercè MARQUÈs KROM, s. 1. restauracio

Màrius VENDRELL, Grup Patrimoni-UB, universitat de Barcelona

Pilar GIRADLES, Grup Patrimoni-UB, universitat de Barcelona

Jordi CAMPS, Sòria Museu Nacional d'Art de Catalunya (MNAC)

Floréal DANIEL, Institut de recherche sur les archéomatériaux (IRAMAT), Centre de recherche en physique appliquée à l'archéologie (CRPAA), université Bordeaux-3

Juliette ROLLIER, université de Bourgogne

José Ramon GARCIA, José Luis ABAD, Antique, s. l.

Anna LLUVERAS, Grup Patrimoni-UB, universitat de Barcelona

Sarah BOULARAND, Grup Patrimoni-UB, universitat de Barcelona

Bàrbara LABORDE, Grup Patrimoni-UB, universitat de Barcelona

Cécile VOYER, université Michel de Montaigne/Bordeaux-3, Centre d'études supérieures de civilisation médiévale de Poitiers

Clara PAYÀs, Taller de restauració Art mural

Juan Antonio olAÑETA, Licenciado en Ciencias Empresariales. Miembro fundador de Amigos del Románico y creador de la página web www.claustro.com

Pascal MORA, Archéotransfert. Institut Ausonius, université de Bordeaux 
INDEX

Mots-clés : art roman, iconographie, matériau, peinture murale, polychromie, technique de construction

Index géographique : France/Pyrénées 